12 Annual Conference of the Materials Research Society of Serbia, Herceg Novi, Montenegro, September 6-10, 2010

\title{
Influence of the Nanostructures on the Surface and Bulk Physical Properties of Materials
}

\author{
N.V. Kamanina ${ }^{a, b, c}$, N.A. Shurpo ${ }^{a}$, S.V. Likhomanova ${ }^{c}$, S.V. Serov ${ }^{a}$, P.Ya. Vasilyev $^{a}$, \\ V.G. Pogareva ${ }^{a}$, V.I. Studenov ${ }^{a}$, D.P. UskokoviC ${ }^{d}$ \\ ${ }^{a}$ Department of Photophysics of media with nanoobjects, Vavilov State Optical Institute, 12, Birzhevaya Line, St. \\ Petersburg, 199034, Russia \\ ${ }^{b}$ Electrotechnical University, 5, Professor Popov str., St. Petersburg, 197376, Russia \\ ${ }^{c}$ St.-Petersburg University of Fine Mechanics and Optics, Kronverkskii Pr., 49, St. Petersburg, 197101, Russia \\ ${ }^{d}$ Institute of Technical Sciences of the Serbian Academy of Sciences and Arts, Knez-Mihailova 35/IV, Belgrade, \\ 11000, Serbia
}

\begin{abstract}
Fullerenes, nanotubes, quantum dots are considered as effective sensitizers to modify both the optical, nonlinear optical features, dynamic and polarization characteristics, as well as mechanical and spectral properties of the organic and inorganic materials. The correlation between photorefractivity and photoconductivity was supported and the relation between charge carrier mobility of pure conjugated structures and nanoobjects-doped ones has been revealed. An increase of transmission of nanostructured polarization films was observed. An extension of the nanocomposites applications area is considered.
\end{abstract}

PACS: 42.70.-a, 42.79.--e, 78.20.-e, 78.30.Na

\section{Introduction}

The properties of nanostructured materials and related phenomena were extensively studied during the last decade. Fullerenes, carbon nanotubes (CNTs), quantum dots (QDs, for example, such as $\mathrm{CdSe}(\mathrm{ZnS})$, etc.) were considered as new effective sensitizers. These investigations open new directions to study the bulk and surface properties for basic research and determine promising fields for practical applications of these systems [1, 2].

In studying the spectral, photorefractive, photoconductive properties of the organic materials and the mechanisms responsible for these phenomena, it was established that the main advantages of modifying the bulk properties by doping with fullerenes and quantum dots are the unique system of energy levels and a relatively high electron affinity, while the important properties of CNTs are their high conductivity and the variety of charge transfer mechanisms. Previously, we demonstrated [3-5] the formation of barrier-free charge transfer pathways, increased dipole moment, and increased specific (per unit volume) local polarizability in some organic matrices doped with fullerenes, carbon nanotubes and quantum dots, where the formation of intermolecular complexes predominates over the intramolecular donor-acceptor interaction. The possible schemes of charge transfer via different nanosensitizers and organic molecule donor fragment are shown schematically in Fig. 1.

Analyzing the data shown in Fig. 1, one can say that when emphasis is made on fullerene or quantum dots (for example, such as $\mathrm{CdSe} / \mathrm{ZnS}$ ) doping, it is neces- sary to take into account the charge transfer between organic molecule donor fragment and nanosensitizers due to their high electron affinity energy $(2.65-4.5 \mathrm{eV})$ that is more than the ones for intramolecular acceptor fragments. When the organics molecules are sensitized by CNTs, it is necessary to take into account the variety of charge transfer pathways, including those along and across a CNT, between CNTs, inside a multiwall CNT, between organic molecules and CNTs, and between the donor and acceptor moieties of an organic matrix molecules.

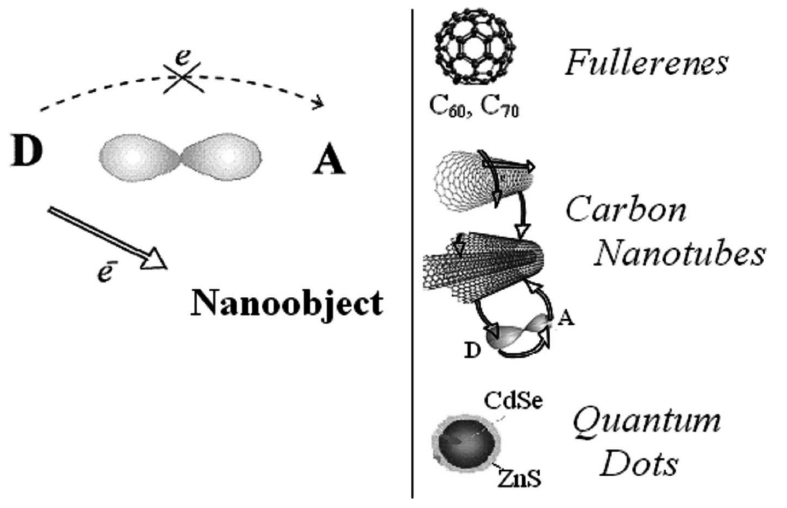

Fig. 1. Model consideration of the efficient charge transfer via nanoobjects doping.

In studying the surface and mechanical properties of the organic and inorganic materials the unique features of the carbon nanotubes, which modify the materials sur- 
face with large advantage, should be taken into account Really, firstly, a little value of the refractive index of the carbon nanotubes, $n \sim 1.1$, permits to develop the effective intermediate layer between air and matrix materials. Secondly, the carbon nanotubes imaginary part of the dielectric constant is close to zero in the near and middle infrared spectral range that permits to save or improve the spectral properties of the matrix materials after nanoobjects placement on the surface. Thirdly, due to large hardness of $\mathrm{C}-\mathrm{C}$ bonds in carbon nanotubes, of the order TeraPascal $[6,7]$, these nanoobjects improve the surface and bulk hardness of matrix materials and decrease the surface roughness.

\section{Experimental conditions}

To reveal a modification of bulk properties, the organic thin films based on $\pi$-conjugated polyimide (PI) sensitized with fullerenes, nanotubes or quantum dots have been chosen for the study. In addition, a nematic liquid crystal (NLC) was also used as an organic matrix. The concentration of fullerenes and quantum dots was varied in the range of $0.003-1.0$ wt. $\%$, the ones of carbon nanotubes was in the range of $0.01-0.1 \mathrm{wt} . \%$, The films were obtained by using the centrifuge deposition. The thickness of the films was $2-5$ micrometers. The LC cell thickness was 5-10 micrometers. The nanostructured LC films were placed onto glass substrates covered with transparent conducting layers based on ITO contacts. The nanostructured monomer or polymer films were deposited on the substrate with ITO contact. For the electric measurements, gold contact was put to the polymer upper side. The bias voltage applied to the photosensitive polymer layers has been varied from 0 to $50 \mathrm{~V}$. The current-voltage characteristics were measured under the illumination conditions from dark to light illumination. Voltmeter-electrometer B7-30 and Characteriscope-Z, type TR-4805 was used for these photoconductive experiments. The photorefractive characteristics have been studied using four-wave mixing technique analogous to paper Ref. [8]. The second harmonic of pulsed Nd-laser at wave length of $532 \mathrm{~nm}$ has been used. The laser energy density has been chosen in the range of $0.1-0.5 \mathrm{~J} \mathrm{~cm}^{-2}$. The nanosecond laser regime (the pulse width was 10$20 \mathrm{~ns})$ was applied. The amplitude-phase thin gratings were recorded under Raman-Nath diffraction conditions at spatial frequency of $90-150 \mathrm{~mm}^{-1}$.

To reveal the surface properties modification of the materials via carbon nanotubes placement, CNTs were laser deposited onto the surface of optical materials or polarization films in vacuum using $p$-polarized radiation of a quasi $\mathrm{CW} \mathrm{CO}_{2}$ laser. During the deposition, the CNTs were oriented by applying an electric field with strength of $50-200 \mathrm{~V} / \mathrm{cm}$. The optical transmission measurements were performed using an SF-26 spectrophotometer operating at 200-1200 nm wavelength range and using Perkin Elmer Lambda 9 spectral device for UV and IR study. The results of spectral measurements were checked using calibrated optical filters. The error in transmission measurements did not exceed $0.2 \%$.

\section{Results and discussion}

\subsection{Bulk properties}

Table I represents the current-voltage characteristics of some pure and nanosensitized compounds. The basic accent was given on conjugared PIs. It should be mentioned that the current-voltage characteristics of pure and nanoobjects-doped PIs were measured for the samples with various concentrations of fullerene $\mathrm{C}_{70}$ or $\mathrm{C}_{60}$ additives, nanotubes and quantum dots under the conditions of dark and light illumination. Table I presents the current-voltage characteristics data under light irradiation.

One can see from the Table I that the increase in the current response can be observed after fullerene sensitization. This fact is connected with the charge transfer complex (CTC) formation between donor part of the organic molecule and fullerene. To support the CTC formation, the mass-spectrometry analysis has been made. The mass spectroscopy data point to the CTC formation between fullerene and triphenylamine in the $\mathrm{C}_{70}$-PI systems [9]. Photoconductive measuring shows that an increase in photoconductivity by at least two orders of magnitude is observed for all fullerene-doped films. Therefore, photoconductivity and mass spectrometry data confirm the complex formation in the studied structures. Regarding the nanotubes-sensitized PI compounds, it should be mentioned, that the fact of dramatical increase in photocurrent can be explained by the additional complex mechanisms responsible for the electron moving in the conjugated organics with nanotubes. The additional odd electron from the core of the nanotubes should be taken into consideration. More than six orders of magnitude difference in the current were observed at the same value of bias voltage. These data are in good coinciding with the data obtained in the paper Ref. [10]. Increase of current response has been found in PI doped with little content of QDs based on the system of CdSe/ZnS.

Some procedure to estimate the charge currier mobility for the pure, fullerene-, nanotubes-, and quantum dotsdoped PI has been made. It should be noticed that for PI conjugated systems we have drown the attention mostly on the role of charge carrier mobility rather than on their concentration. This is explained by the fact that in this type of organics the conductivity activation energy coincides with the mobility activation energy, plotted in the double logarithmic scale, the values of conductivity and mobility (measured at equal temperatures and electric field strengths) fit to the same straight line inclined at $45^{\circ}$ relative to the coordinate axes. The charge carrier mobility was estimated via the Child-Langmuir currentvoltage relationship by the method proposed in paper Refs. $[11,12]$. The charge carrier mobility of pure and nanoobjects-doped samples were estimated at the same density of the current, bias voltage, thickness of the films, and dielectric permittivity. The following relationship was found:

$\mu_{\text {pure }}<\mu_{\text {fullerenes }}<\mu_{\mathrm{NT}, \mathrm{QD}}$. 
TABLE I

Photocurrent of polyimides with different nanoobjects.

\begin{tabular}{l|c|c|c|c}
\hline \hline Bias voltage & \multicolumn{3}{|c}{ Current, A, under light irradiation } \\
$\mathrm{V}$ & Pure PI & PI +0.2 wt.\% C70 & PI +0.1 wt.\% CNT & PI +0.003 wt.\% QD \\
\hline 0 & $2.77 \times 10^{-12}$ & $4.33 \times 10^{-12}$ & & \\
1 & & & Close to $10^{-4}-10^{-3}$ & \\
5 & $2.98 \times 10^{-11}$ & $5.0 \times 10^{-11}$ & & \\
10 & $6.96 \times 10^{-11}$ & $1.1 \times 10^{-10}$ & & \\
15 & $1.01 \times 10^{-10}$ & $2.3 \times 10^{-10}$ & & Close to $10^{-9}-10^{-8}$ \\
20 & $1.44 \times 10^{-10}$ & $3.7 \times 10^{-10}$ & & \\
30 & $2.5 \times 10^{-10}$ & $8.0 \times 10^{-10}$ & & \\
40 & $3.8 \times 10^{-10}$ & $1.4 \times 10^{-9}$ & & \\
50 & $5.7 \times 10^{-10}$ & $2.4 \times 10^{-9}$ & &
\end{tabular}

TABLE II

Laser-induced change of the refractive index.

\begin{tabular}{l|c|c|c|c|c}
\hline \hline Structure & $\begin{array}{c}\text { Nanoobjects } \\
\text { content, wt\% }\end{array}$ & $\begin{array}{c}\text { Energy density } \\
\mathrm{J} \mathrm{cm}^{-2}\end{array}$ & $\begin{array}{c}\Lambda \\
\mathrm{mm}^{-1}\end{array}$ & $\Delta n$ & Ref. \\
\hline Pure PI & 0 & 0.6 & 90 & $10^{-4} \div 10^{-5}$ & {$[13]$} \\
PI + quantum dots based on CdSe(ZnS) & 0.003 & $0.2-0.3$ & 100 & $2.0 \times 10^{-3}$ & current \\
PI+C70 & 0.2 & $0.5-0.6$ & 90 & $4.68 \times 10^{-3}$ & {$[13]$} \\
PI + nanotubes & 0.1 & $0.3-0.5$ & $100-150$ & $5.5-5.7 \times 10^{-3}$ & {$[13]$} \\
Polymer-dispersed LC based on PI-C70 complex & 0.2 & $0.1-0.3$ & $90-100$ & $1.2 \times 10^{-3}$ & current
\end{tabular}

The results of these calculations show that the introduction of fullerenes leads to a tenfold increase in the mobility, but introduction of nanotubes or quantum dots reveals increase of mobility by two or more orders of magnitude. Thus, charge carrier mobility of pure PI films placed in the interval from $10^{-7}$ to $10^{-5} \mathrm{~cm}^{2} \mathrm{~V}^{-1} \mathrm{~S}^{-1}$ can be increased to $10^{-4}-10^{-3} \mathrm{~cm}^{2} \mathrm{~V}^{-1} \mathrm{~S}^{-1}$.

This fact correlates with increase of dynamic properties of the nanostructures-doped conjugated films and liquid crystal cells [3]. It should be noticed that at smaller quantum dots concentration than nanotubes concentration, the charge carrier mobility has been found a little bit larger for QD-doped structures in comparison with nanotubes-doped ones and at smaller nanoobjects concentration, the charge carrier mobility has been found a little bit larger for nanotube-doped structures in comparison with fullerene-doped ones.

It should be noticed that the photoconductive parameters coincide with the photorefractive ones. Really, for polyimide matrixes the basic results regarded to the laser-induced change of refractive index, $\Delta n$, under condition of different nanoobjects sensitization and at varied spatial frequency, $\Lambda$, are shown in Table II.

It should be taken into account that for fullerenedoped PI irradiating laser energy density $W_{\text {in }}$ of $0.5-0.6$ $\mathrm{J} \mathrm{cm}^{-2}$ corresponds to $350-400 \mathrm{kcal} \mathrm{mol}^{-1}$ at molecular mass of PI units of 750 . The rotation threshold is
100-700 kcal mol ${ }^{-1}$ [14]. This effect is likely to result in a better arrangement of PI donor fragment (triphenylamine) and fullerene planes, and hence in efficient CT between them [15].

From the laser-induced changes of the refractive index, the nonlinear refraction $n_{2}$ and the third order susceptibility $\chi^{(3)}$ were determined via procedure shown in the paper. [13]. They were, respectively: $\sim 10^{-7} \mathrm{~cm}^{2} \mathrm{~kW}^{-1}$ and $\sim 10^{-9}$ esu for thin conjugated films of the nanoobjects-doped organic structures, $\sim 10^{-6} \mathrm{~cm}^{2} \mathrm{~kW}^{-1}$ and $\sim 10^{8}$ esu for thin films of the nanoobjects-doped polymer-dispersed liquid crystals. The model explaining improving of nonlinear optical and of dynamicn features of the nanoobjects-doped organic structures via increase in dipole moment and local volume polarizability were firstly early presented in the paper Ref. 3 . It has been postulated that the same evidences have been found for the structures based on 2-cyclooctylamino-5nitropyridine (COANP), $N$-(4-nitrophenyl)- $(L)$-prolinol (NPP), polyanilyne (PANI) doped with nanoobjects.

It should be mentioned that nonlinear optical parameters of nanoobjects-doped structures are larger than those obtained for traditional nonlinear inorganic systems and for pure organics. Moreover, the data testify that nonlinear characteristics of the materials studied are close to those for Si-based structures and larger than the ones for classical inorganic widely used crystal of $\mathrm{LiNbO}_{3}$, 
that provoke the organics structures with nanoobjects to be used in extended area of optical and non-linear optical applications.

\subsection{Surface properties}

Unique influence of the nanoobjects placement on surface mechanical hardness and roughness of the soft materials of UV an IR range has been shown in paper Ref. 16. As new aspect of this study the nanoobjects structurization of inorganic systems has been found for polyvinyl alcohol (PVA) iodine films used as polarizators in display and telecommunications schemes.

We have studied polarizers, such as iodinated PVA films with a thickness of $60-80 \mu \mathrm{m}$, which were coated on both sides by $\sim 0.05 \mu \mathrm{m}$ thick layers of single walled CNTs.

The polarizers contained polarization films with either parallel or mutually perpendicular (crossed) orientations, depending on the need to obtain the initial bright or dark field. It was found that the modification (nanostructuring) of the surface of polarization films by CNTs led to some increase in the optical transmission for the parallel component of incident light (see curves 1 and 2 in the Fig. 2), while retaining minimum transmission for the orthogonal component (see curves 3 and 4 in Fig. 2).

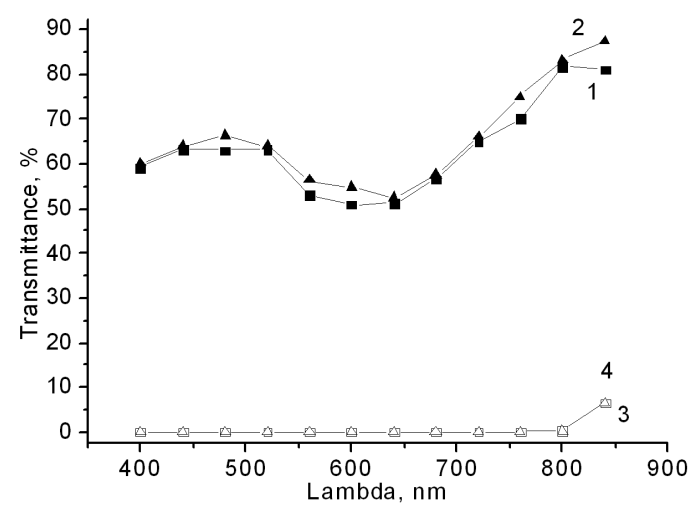

Fig. 2. Transmittance for the parallel $(1,2)$ and orthogonal $(3,4)$ part of light before $(1,3)$ and after $(2,4)$ nanotreatment procedure of the polarizer film.

\section{Conclusions}

The influence of nanoobjects, based on fullerenes, carbon nanotubes and quantum dots, on bulk and surface physical properties of optical materials was studied and the observed results are discussed here. The correlation between photorefractivity and photoconductivity has been considered as the feature of nanoobjectssensitized organics. The relation between charge carrier mobility of pure conjugated structures and nanoobjectsdoped ones has been revealed. The nonlinear optical parameters, such as nonlinear refraction and cubic nonlinearity, for nanoobjects-doped systems were calculated and the place of them among nonlinear characteristics of traditional nonlinear materials was postulated. Moreover, as new vision, the surface modification of polar- ization films via nanostructurisation procedure was revealed. The nanostructuring of the surface of polarization films by CNTs led to a $2-5 \%$ increase in the optical transmission in the visible spectral range for the parallel component of incident light, while retaining minimum transmission for the orthogonal component. As the results of investigations, the potential area of applications of the materials is in optoelectronics and laser optic. Examples of possible applications are: telecommunication systems, gas storage and solar energy accumulation, as well as in the field of nonlinear optics and in search for 3D-media and new display elements.

\section{Acknowledgments}

The authors would like to thank their colleague Prof. N. M. Shmidt (Ioffe Physical-Technical Institute, St.Petersburg) for their help in study of photoconductive characteristics. The work is supported by Russian Foundation for Basic Researches (grant 10-03-00916, 20102012, travel grant 10-03-08190, 2010) and by Vavilov State Optical Institute (grant "Perspectiva", 2010).

\section{References}

[1] S. Couris, E. Koudoumas, A.A. Ruth, S. Leach, J. Phys. B: At. Mol. Opt. Phys. 8, 4537 (1995).

[2] J. Robertson, Mater. Today 7, 46 (2004).

[3] N.V. Kamanina, Physics-Uspekhi 48, 419 (2005).

[4] N.V. Kamanina, S.V. Serov, V.P. Savinov, Tech. Phys. Lett. 36, 40 (2010).

[5] N.A. Shurpo, M.S. Vakshtein, N.V. Kamanina, Tech. Phys. Lett. 36, 319 (2010).

[6] S. Namilae, N. Chandra, C. Shet, Chem. Phys. Lett. 387, 247 (2004).

[7] M.M.J. Treacy, T.M. Ebbessen, J.M. Gibson, Nature 381, 678 (1996).

[8] N.V. Kamanina, N.A. Vasilenko, Opt. Quantum Electron. 29, 1 (1997).

[9] N.V. Kamanina, A.I. Plekhanov, Optics and Spectroscopy 93, 408 (2002).

[10] Xiaowen Jiang, Yuezhen Bin, Masaru Matsuo, Journal Polymer 46, 7418 (2005).

[11] F. Gutman, L.E. Lyons, Organic Semiconductors, Wiley, New York 1967.

[12] M.M. Mikhailova, M.M. Kosyreva, N.V. Kamanina, Technical Physics Letters, 28, 450 (2002).

[13] N.V. Kamanina, S.V. Serov, V.P. Savinov, D.P. Uskoković, Int. J. Mod. Phys. B), 24, 695 (2010).

[14] V. Bershtein, V. Egorov, DSC in Physical Chemistry of Polymers, Chemistry, Leningrad 1990.

[15] Y.A. Cherkasov, N.V. Kamanina, E.L. Alexandrova, V.I. Berendyaev, N.A. Vasilenko, B.V. Kotov, Proceed. SPIE 3471, 254 (1998).

[16] N.V. Kamanina, P.Ya. Vasilyev, S.V. Serov, V.P. Savinov, K.Yu. Bogdanov, D.P. Uskokovic, Acta Phys. Pol. A, 117, 786 (2010). 